\title{
Nutritional Challenges of Children with Disabilities in Special Needs Center in Mainland, Lagos State, Nigeria
}

\author{
Kemi Priscillia Ogbonna ${ }^{1}$, Victoria Omonigho Otobo ${ }^{2}$, Felicia Obiageli Maduabum ${ }^{1}$, Francisca Nonyelum \\ Onyeka ${ }^{1}$, Olatokunboh Ayodele Ashiru ${ }^{3}$ and Chika Esther Ajuluchi ${ }^{4}$ \\ 1. Department of Home Economics \& Hospitality Management Education, Faculty of Vocational \& Technical Education, University \\ of Nigeria, Nsukka 410001, Nigeria \\ 2. Home Economics Department, School of Vocational Education, Federal College of Education (Technical), Akoka 100001, Yaba, \\ Lagos State, Nigeria \\ 3. Department of Fashion Design, School of Art Design and Printing, Yaba College of Technology, Lagos 101212, Nigeria \\ 4. Department of Home Economics, Federal College of Education, Okene 260101, Kogi State, Nigeria
}

\begin{abstract}
The study determined the nutritional challenges of persons with disability in special needs centers in mainland, Lagos State. Three research questions guided the study. Survey research design was adopted. The population of the study was 57 . No sampling was done since the population was a manageable size. Validated questionnaire was used for data collection. Data collected were analyzed using mean and standard deviation. Findings showed that the types of disabilities among children in special need centers in Lagos State included among others: physical disability, learning disability, cerebral palsy, Down syndrome, attention deficit disorder/attention deficit hyperactivity disorder (ADHD), autism and speech and language disorder. Findings also revealed that the nutritional needs of children with disability included feeding on different foods, drinking water regularly, taking fruits daily, and ensuring that their nutrient requirements are met daily. Findings indicated that the nutritional challenges of children with disability included among others: excessive salivation, frequent constipation, inability to feed oneself, depending on care givers for feeding, food dropping from the mouth while being fed and difficulty in chewing and swallowing foods. Conclusion was made. Among recommendations made was that children with disabilities should be provided with variety of foods daily to meet their nutritional needs and parents/caregivers should devote more feeding time to the disabled child.
\end{abstract}

Key words: Nutrition, challenges, disabilities, children, special needs.

\section{Introduction}

There is a global trend towards eating healthier foods and improving nutrition. Adequate nutrition entails taking of diet that contains all the essential food nutrients. Mela [1] reported that adequate nutrition is a diet that provides all the essential nutrients and calories needed to maintain good health and acceptable body weight. During childhood and adolescence, adequate nutrition is important to achieve full growth potential and appropriate body composition, to promote health

Corresponding author: Kemi Priscillia Ogbonna, MSc (Food \& Nutrition), research fields: food \& nutrition (food security, food safety/hygiene). and well-being, and to reduce the risk of chronic diseases in adulthood. Children require sufficient energy, protein, and other nutrients for growth as well as maintenance of body functions. Nutrient needs tend to lie in parallel rates of children growth. Olusanya [2] noted that growth continues at a steady rate during childhood then, accelerates during adolescence, creating increases in nutrient needs to support the rapid growth rate and increase in lean body mass and body size. The nutrient requirements among individuals differ with age, gender, body size and physiological state. Each nutrient performs different functions in individuals' body.

When nutrients are not consumed in quantities 
commensurate with individuals' body needs or excessively consumed, it may lead to malnutrition or over-nutrition. World Health Organisation (WHO) [3] reported that malnutrition is a condition that results from eating a diet in which one or more nutrients are either not enough or are too much so that the diet causes health problems. In addition to the impact of adequate nutrition on child's growth and development, children's diets are important to ensure overall health and well-being. Tuzun et al. [4] reported that a high incidence/prevalence of malnutrition is often reported in children with disability, and this may result in poorer health and development, leading to a perpetuating cycle of sub-optimal nutrition, disability and worsening health status. Hence, there are nutritional challenges faced by children with disabilities.

Disability is an umbrella term, covering impairments, activity limitations, and participation restrictions. According to World Health Organization [5], a disability is an impairment that may be cognitive, developmental, intellectual, mental, physical, sensory, or some combination of these. Impairment is a problem in body function or structure; an activity limitation is a difficulty encountered by an individual in executing a task or action, while a participation restriction is a problem experienced by an individual in involvement in life situations. Americans with Disabilities Act (ADA) [6] stated that disability means a physical or mental impairment that substantially limits one or more of the major life activities. Disability is thus not just a health problem. It is a complex phenomenon, reflecting the interaction between features of a person's body and features of the society in which he or she lives. There are so many disabilities that can trouble children's lives and in so many cases they are not even identified as they are simply looked at as bad behaviour. ADA [6] reported that disabilities among children can be categorized into physical impairment which includes but is not limited to asthma, blindness, deafness, seizures, heart disease, or cerebral palsy; mental impairment which includes but is not limited to developmental delay, behaviour disorders, or learning disabilities and major life activities which include breathing, hearing, seeing, speaking, walking, using of arms and legs, learning and playing.

Given opportunities to flourish as others might, children with disabilities have the potential to lead fulfilling lives and to contribute to the social, cultural and economic vitality of their communities. Yet surviving and thriving can be especially difficult for children with disabilities. Hakime [7] pointed out that there are wide-ranging challenges that are integral to parenting a child with disability. According to Bhutta et al. [8] children with disabilities generally require more attention because they may have additional needs when compared to nondisabled children. Maulik and Darmstadt [9] mentioned that parents of children with disability may experience constant subjection to a guilty feeling that they may be directly responsible for the disability through genetics, stressful moments while pregnant or abuse of alcohol. The right to food is enshrined into various Human Rights charters; however the nutritional needs of children with disabilities and special needs are rarely addressed. A special needs child is one who has been determined to require special attention and specific necessities that other children do not. Kajan [10] stated that special need is an umbrella term for a wide array of diagnoses, from those that resolve quickly to those that will be a challenge for life and those that are relatively mild to those that are profound. It covers developmental delays, medical conditions, psychiatric conditions, and congenital conditions that require accommodations so children can reach their potentials. $\mathrm{Tu}$ and Cunningham [11] reported that special needs can range from people with autism, cerebral palsy, Down syndrome, dyslexia, blindness, attention deficit hyperactivity disorder (ADHD), and cystic fibrosis.

Children with special needs may have mild learning disabilities or profound cognitive impairment, terminal illness, developmental delays and occasional panics [12]. Children with learning disabilities often lack the 
ability to articulate personal strengths and needs clearly and appropriately. This is as a result of expressive language difficulties, weak social skills or lack of practice in describing their needs. Many children with disabilities have health issues that can impact their nutritional well-being and eating habits. Groce et al. [13] reported that some children with disabilities may need additional nutrients to cope with the health problems associated with their disability. For example, a child with a physical disability may be prone to pressure sores caused by immobility or poor nursing which can become seriously infected. A high-quality diet is needed for prompt healing and to control infection.

Although the focus of concern for children with disability is generally around under-nutrition, becoming overweight is also a concern for some disabled children. For example, children with certain physical disabilities are less mobile and therefore at risk of becoming overweight [2]. Other children with certain types of genetic impairments (e.g., Sotos syndrome) or intellectual or mental health disabilities may have eating disorders which place them at greater risk of becoming overweight. Overweight does not mean that all parts of the diet exceed normal needs: micronutrient deficiencies can occur in a high-energy but poor-quality diet and go unnoticed by parents and caregivers, with serious long-term consequences for health and development.

Children with disabilities rely more on family members and care givers for food thereby placing severe time constraints and economic burdens on the caregivers. Parents and caregivers may lack knowledge of how to feed their disabled child effectively or of how to teach the child to feed himself. This is especially important for children with conditions such as cerebral palsy who may need special seating or positioning to control muscle spasms or for children with Down's syndrome who are at increased risk of choking and developing pneumonia. Difficult meal times can lead to increased stress levels for the caregiver and the child, which can result in insufficient food intake [14]. The burden of feeding children with disabilities is also a gender issue as the responsibility of feeding such children falls most heavily on female members of the household who must balance the needs of the child with all other necessary chores. The result is that the child may receive less food because it is time-consuming to feed a child with low muscle tone and spasticity and no-one in the household has the time or energy to provide such feeding. This is an issue not only in the home. The UK-based international NGO Lumos [15] reported that, owing to time and work constraints, caretakers of children with disabilities in Bulgarian institutions were unable to devote more than 2 minutes to each child during meal-times, despite the fact that these children needed help with feeding and drinking.

A child whose disability or developmental delay is identified at an early stage will have a much better chance of reaching her or his full capacity. Children with disabilities require care from either their parents or care givers in a child care center. In a childcare center, teachers focus on the physical and mental developments of their students. In order to have a greater understanding of the student, teachers in centers must incorporate a relationship with their students that benefit their wants and needs while pushing to enable them to meet their needs. Childcare is seen as a reasonable option because it is different from parenting, since it can be seen as more of a routine for the child [16]. Children are placed into centers of socialization where they learn many similarities and differences from one another from a very young age. Children are also placed into settings to develop their linguistics and cognitive abilities, which can be measured through observations [16].

Childcare centers provide different experiences than that parental care does, especially when there is availability of adequate professionally trained child care experts. However, observation revealed that there is paucity of trained specialists to handle special 
children in the childcare centers. Right now there is a dearth of teachers especially those trained to attend to the needs of special children. The challenges of catering for the nutritional needs of the special children are many and varied. Groce et al. [13] reported that most special-needs children have difficulty in swallowing food, expelling excessive salivation; they experience frequent constipation and sometimes food drop from the mouth while being fed. This implies that majority of the special-needs children in childcare centers depend on the caregivers for realizing their nutritional requirements. It was based on this backdrop that the study was undertaken to determine the nutritional challenges of children with disabilities in special needs centers in mainland, Lagos State, Nigeria.

\section{Purpose of the Study}

The main purpose of the study was to determine the nutritional challenges of children with disability in a special need center in mainland, Lagos State.

Specifically, the study determined:

(1) types of disabilities among children in a special need center in Mainland, Lagos State;

(2) nutritional needs of children with disabilities in a special need center in Mainland, Lagos State;

(3) nutritional challenges of children with disabilities in a special need center in Mainland, Lagos State.

\section{Research Questions}

The following research questions guided the study:

(1) What are the types of disabilities among children in a special need center in Mainland, Lagos State?

(2) What are the nutritional needs of children with disabilities in a special need center in Mainland, Lagos State?

(3) What are the nutritional challenges of children with disabilities in a special need center in Mainland, Lagos State?

\section{Methodology}

\subsection{Design of the Study}

Survey research design was adopted for the study.

\subsection{Area of the Study}

The study was conducted in Mainland, Lagos State. Mainland, Lagos State was chosen for the study because it is an urban area with large number of special needs centers. However, the study was conducted in one special need center; Modupe Cole Memorial Child Care and Treatment Home School situated at Oluwole Street, off St. Finbarr's Road, Akoka, Lagos Mainland in Lagos State, Nigeria.

\subsection{Population for the Study}

Population of the study was fifty-seven (57). This comprised of care givers in a special need center situated in Mainland, Lagos State.

\subsection{Sample and Sampling Techniques}

No sampling was done since the population was a manageable size.

\subsection{Instrument for Data Collection}

The instrument used for data collection was a structured questionnaire. The instrument was titled "Nutritional Challenges of Children with Disabilities in Special Need Center (NCPDSNC)”. It consisted of two sections. Section A elicited information on socio demographic characteristics of the respondents while Section B was based on the purposes of the study. The structured questionnaire was coded with nominal values which are assigned to each possible response expected from the respondents. A four-point scale rated as follows was used: Strongly Agreed, Agreed, Disagreed, and Strongly Disagreed.

\subsection{Validation of Research Instrument}

This was determined by test re-test method. The 
questionnaire was pretested to 20 care givers at Morgan Hill Children Foundation Ikeja. The center is outside the local government of the research area. The questionnaire was tested again after two weeks. A reliability co-efficient of 0.87 was obtained using spearman's correlation method.

\subsection{Ethical Considerations}

Ethics approval was obtained from the management of the special needs centers. Written informed consent was obtained from all caregivers used for the study.

\subsection{Method of Data Collection}

The instrument was administered to the care givers and retrieved on the spot by the researchers. The care givers responded to the questionnaires. Efforts were made to ensure that the items were filled correctly without omitting any of the needed information.

\subsection{Method of Data Analysis}

The data collected were analyzed using percentage, mean and standard deviation. Mean rating from 2.5 and above was considered agreed upon while mean rating of 2.49 and below was considered as disagreed upon.

\section{Results}

Table 1 showed that all but one of the listed types of disabilities among children in a special need center were agreed upon. The mean values ranged from 2.55 to 3.89. However, item 11 was disagreed upon. This means that few of the special-needs children were blind. On the other hand, the standard deviation ranged from 0.64 to 1.02 implying that the mean vales were close to each other.

Table 2 showed that all the listed items on nutritional needs of children with disability in special needs centers were all accepted. The mean values ranged from 2.90 to 3.90 which are above the cutoff point of 2.50. Also, the standard deviation ranged from 0.78 to 1.01 indicating that the mean values were close to each other.

Table 3 indicated that all the highlighted items were agreed upon as nutritional challenges of children with disability in special needs centers. The mean values ranged from 2.53 to 4.00 . The standard deviation ranged from 0.75 to 1.08 implying that the mean responses were close to each other.

Table 1 Mean and standard deviation responses on types of disabilities among children in childcare centers in Mainland, Lagos State.

\begin{tabular}{lllll}
\hline S/N & Types of disabilities among children in childcare centers & X & SD & Remark \\
\hline $\mathbf{1}$ & Physical disability & 3.29 & 1.02 & Agree \\
2 & Learning disability & 3.08 & 1.06 & Agree \\
3 & Cerebral palsy & 3.86 & 0.69 & Agree \\
4 & Down syndrome & 3.89 & 0.90 & Agree \\
5 & Attention deficit disorder/attention deficit hyperactivity disorder & 3.10 & 0.97 & Agree \\
6 & Dyslexia & 2.86 & 0.64 & Agree \\
7 & Asperger's syndrome or autism & 2.59 & 0.91 & Agree \\
8 & Central auditory processing disorder & 2.88 & 0.82 & Agree \\
9 & Aphasia & 2.55 & 0.90 & Agree \\
10 & Behavior disorder & 3.88 & 0.96 & Agree \\
11 & Blindness & 1.50 & 0.81 & Disagree \\
12 & Deafness & 2.58 & 0.75 & Agree \\
13 & Partial deafness & 3.02 & 0.83 & Agree \\
14 & Speech and language disorder & 3.79 & 0.84 & Agree \\
\hline
\end{tabular}


Table 2 Mean and standard deviation responses on nutritional needs of children with disability in special need center in Mainland, Lagos State.

\begin{tabular}{|c|c|c|c|c|}
\hline $\mathrm{S} / \mathrm{N}$ & Nutritional needs of persons with disability & $\mathrm{X}$ & $\mathrm{SD}$ & Remark \\
\hline 1 & Feeding on different foods & 3.58 & 0.85 & Agree \\
\hline 2 & Drinking water regularly & 3.77 & 1.01 & Agree \\
\hline 3 & Taking fruits daily & 3.90 & 0.99 & Agree \\
\hline 4 & Eating adequate meals always & 3.88 & 0.85 & Agree \\
\hline 5 & Avoiding skipping of meals & 2.90 & 0.78 & Agree \\
\hline 6 & Feeding at regular intervals & 3.47 & 0.91 & Agree \\
\hline 7 & Ensuring that their nutrient requirements are met daily & 3.80 & 0.82 & Agree \\
\hline
\end{tabular}

Table 3 Mean and standard deviation responses on nutritional challenges of children with disability in special need center in Mainland, Lagos State.

\begin{tabular}{lllll}
\hline S/N & Nutritional challenges of persons with disability & $\mathrm{X}$ & SD & Remark \\
\hline 1 & Frequent eating & 3.13 & 1.08 & Agree \\
2 & Excessive salivation & 3.72 & 0.82 & Agree \\
3 & Frequent constipation & 2.90 & 0.92 & Agree \\
4 & Inability to feed oneself & 3.89 & 1.06 & Agree \\
5 & Depends on care givers for feeding & 4.00 & 1.00 & Agree \\
6 & Food dropping from the mouth while being fed & 2.50 & 0.75 & Agree \\
7 & Difficulty in swallowing solid foods & 3.55 & 0.94 & Agree \\
8 & Difficulty in swallowing liquid foods & 2.53 & 0.76 & Agree \\
9 & Difficulty in chewing foods & 2.99 & 1.00 & Agree \\
\hline
\end{tabular}

\section{Discussion of Findings}

Table 1 showed that the types of disabilities among children in childcare center included physical disability, learning disability, cerebral palsy, Down syndrome, attention deficit disorder/attention deficit hyperactivity disorder (ADHD), dyslexia, Asperger's syndrome or autism, central auditory processing disorder, aphasia, behavior disorder, blindness, deafness, partial deafness and speech and language disorder. This implies that there are different types of disabilities among children in special need centers. In support of the findings, WHO [5] stated that special needs children are children who have a disability or a combination of disabilities that makes learning or other activities difficult. These children include those who have mental retardation which causes them to develop more slowly than other children; speech and language impairment such as a problem expressing themselves or understanding others; physical disability such as vision problem cerebral palsy or other conditions; learning disabilities which distort messages from their senses; emotional disabilities such as anti-social or other behavioral problems. In the same vein, Kajan [10] stated that special need is an umbrella term for a wide array of diagnoses, from those that resolve quickly to those that will be a challenge for life and those that are relatively mild to those that are profound. It covers developmental delays, medical conditions, psychiatric conditions, and congenital conditions that require accommodations so children can reach their potentials. Tu and Cunningham [11] reported that special needs can range from people with autism, cerebral palsy, Down syndrome, dyslexia, blindness, ADHD, and cystic fibrosis. Children with special needs may have mild learning disabilities or profound cognitive impairment, terminal illness, developmental delays and occasional panics [12].

Also in line with the findings, ADA [6] reported that disabilities among children can be categorized into physical impairment which includes but is not limited to asthma, blindness, deafness, seizures, heart disease, or cerebral palsy; mental impairment which includes but is not limited to developmental delay, behaviour disorders, or learning disabilities and major life 
activities which include breathing, hearing, seeing, speaking, walking, using of arms and legs, learning and playing.

Findings in Table 2 showed that the nutritional needs of children with disability included feeding on different foods, drinking water regularly, taking fruits daily, eating adequate meals always, avoiding skipping of meals, feeding at regular intervals, and ensuring that their nutrient requirements are met daily. In line with the findings, WHO [3] reported that for optimal development of children, the adequate diet composed of protein, carbohydrate, fats and oils, vitamins and minerals. Groce et al. [13] reported that some children with disabilities may need additional nutrients to cope with the health problems associated with their disability. Also in support of the findings, Mahon and Escott [17] noted that in order to meet the nutritional needs of special children, they need to feed on foods from different sources.

Findings in Table 3 indicated that the nutritional challenges of children with disabilities included frequent eating, excessive salivation, frequent constipation, inability to feed oneself, depending on care givers for feeding, food dropping from the mouth while being fed, difficulty in swallowing solid foods, difficulty in swallowing liquid foods and difficulty in chewing foods. The challenges of catering for the nutritional needs of the special children are many and varied. Groce et. al [13] reported that most special needs children have difficulty in swallowing food, expel excessive salivation, they experience frequent constipation and sometimes food drop from their mouth while being fed. Surviving and thriving can be especially difficult for children with disabilities. Hakime [7] pointed out that there are wide-ranging challenges that are integral to parenting a child with disability. Children with disabilities generally require more attention because they may have additional needs when compared to nondisabled children [8]. Parents and caregivers may lack knowledge of how to feed their disabled child effectively or of how to teach the child to feed himself. This is especially important for children with conditions such as cerebral palsy who may need special seating or positioning to control muscle spasms or for children with Down's syndrome who are at increased risk of choking and developing pneumonia. Difficult meal times can lead to increased stress levels for the caregiver and the child, which can result in insufficient food intake [14]. The result is that the child may receive less food because it is time-consuming to feed a child with low muscle tone and spasticity and no-one in the household has the time or energy to provide such feeding. This is an issue not only in the home but in special needs centers. The UK-based international NGO Lumos [15] reported that, owing to time and work constraints, caregivers of children with disabilities in Bulgarian institutions were unable to devote more than 2 minutes to each child during meal-times, despite the fact that these children needed help with feeding and drinking.

\section{Conclusion}

Special-needs children are those who have a disability or a combination of disabilities that makes learning or other activities difficult. Most of the children depend on parents or caregivers for their feeding. Based on the findings of the study, it was concluded that there are different types of disability among children in special needs centers in Lagos State. Children with disability need additional nutrients to cope with the health problems associated with their disability. The special-needs child also experiences difficult meal times as he/she depends on others for proper feeding.

\section{Recommendations}

Based on the findings of the study, it was recommended that:

(1) Children with disabilities should be provided with adequate diet;

(2) Children with disabilities should be provided with variety of foods daily to meet their nutritional 
needs;

(3) Parents and caregivers should devote more feeding time to the disabled child;

(4) Children with disabilities should be assisted in feeding to ensure that they take in the recommended daily food requirements.

\section{References}

[1] Mela, D. J. 2011. "Determinants of Food Choice: Relationships with Obesity and Weight Control.” Obesity Resources 6 (Supplementary): 249-55.

[2] Olusanya, B. O. 2010. "Is Undernutrition a Risk Factor for Sensorineural Hearing Loss in Early Infancy?” British Journal of Nutrition 103: 1296-301.

[3] World Health Organization (WHO). 2013. "Developmental Difficulties in Early Childhood." Accessed July 16, 2019. https://www.who.int/maternal_child_adolescent/documen ts/development_difficulties_early_childhood/en/.

[4] Tuzun, E. H., Guven, D. K., Eker, L., Elbasan, B., and Bulbul, S. F. 2013. "Nutritional Status of Children with Cerebral Palsy in Turkey.” Disability Rehabilitation 35: 413-7.

[5] World Health Organization (WHO). 2016. "Disabilities.” Accessed July 28, 2019. https//www.WHO_disabilities.org.

[6] American Disability Act (ADA). 2008. "Children with Disability.” Accessed July 15, 2019. https://cshcn.org/resources-contacts/childcare-and-the-ad a/.

[7] Hakime, N. N. 2013. "Nutritional Status in Mentally Disabled Children and Adolescents: A Study from Western Turkey.” Pakistan Journal Medicine Science 29: 614-8.

[8] Bhutta, Z. A., Das, J. K., Rizvi, A., Gaffey, M. F., Walker,
N., and Horton, S. 2013. "Evidence-Based Interventions for Improvement of Maternal and Child Nutrition: What Can Be Done and at What Cost?” Lancet 382: 452-77.

[9] Maulik, P. K., and Darmstadt, G. L. 2007. "Childhood Disability in Low- and Middle-Income Countries: Overview of Screening, Prevention, Services, Legislation, and Epidemiology.” Pediatrics 120 (Suppl. 1): 51-5.

[10] Kajan, J. 2018. "Special Needs Child.” Accessed July 29, 2019. https://www.investopedia.com/terms/s/specialneeds child.asp.

[11] Tu, H. T., and Cunningham, P. J. 2005. "Public Coverage Provides Vital Safety Net for Children with Special Health Care Needs.” Issue on Health System Change 3 (98): 1-7.

[12] Matthews, V. L., Wien, M., and Sabate, J. 2011. "The Risk of Child and Adolescent Overweight.” Nutritional Journal 10 (1): 71-4. Accessed February 10, 2014. http://dx.doi.org/10.1186/1475-2891-10-71.

[13] Groce, N., Challenger, E., Berman-Bieler, R., Farkas, A., Yilmaz, N., Schultink, W., Clark, D., Kaplan, C., and Kerac, M. 2014. "Malnutrition and Disability: Unexplored Opportunities for Collaboration.” Pediatric International Child Health 34 (4): 308-14.

[14] Adams, M. S., Khan, N. Z., Begum, S. A., Wirz, S. L., Hesketh, T., and Pring, T. R. 2012. "Feeding Difficulties in Children with Cerebral Palsy: Low-Cost Caregiver Training in Dhaka, Bangladesh.” Child Care Health Dev 38: 878-88.

[15] Lumos. 2013. "Lumos' Pioneering Training Projects." Accessed July 22, 2019. http://wearelumos.org/stories/lumos-pioneering-trainingprogrammes.

[16] Erel, O., Oberman, Y., and Yirmiya, N. 2000. "Maternal versus Nonmaternal Care and Seven Domains of Children's Development.” Psychological Bulletin 126 (5): 727-47.

[17] Mahan, L. K., and Escott, S. 2008. Foods, Nutrition, \& Diet Therapy. Philadelphia: Saunders Ltd. 